\title{
Usefulness of Sagittal Brain Computed Tomography for Identifying Hyperdense Anterior Cerebral Artery Sign
}

\author{
Jeong-Yoon Lee, MD* (D), Sung-Tae Park, $\mathrm{MD}^{\dagger}$ (D), Hyun-Kyung Lim, $\mathrm{MD}^{\dagger}$ (D), Kyung Bok Lee, MD, PhD ${ }^{*}$ (D) \\ Departments of Neurology ${ }^{\star}$, Radiology ${ }^{\dagger}$, Soonchunhyang University College of Medicine, Seoul, Korea
}

Despite the wide availability of brain magnetic resonance imaging, computed tomography (CT) still remains the imaging modality of choice in acute ischemic stroke. Hyperdense artery sign is the earliest indicator of intracranial occluding clots on non-enhanced brain CT, and has been associated with poor clinical outcomes. While hyperdense middle cerebral artery signs are frequently mentioned, hyperdense anterior cerebral artery (ACA) signs have rarely been reported, especially in the sagittal plane. This study demonstrates a case of hyperdense ACA sign identified on sagittal brain CT followed by successful intravenous thrombolysis.

J Neurosonol Neuroimag 2020;12(1):55-57

Key Words: Stroke; Computed tomography; Anterior cerebral artery; Thrombus; Intravenous thrombolysis

Received: February 17, 2020

Revised: March 23, 2020

Accepted: March 27, 2020

Correspondence:

Kyung Bok Lee, MD, PhD

Department of Neurology, Soonchunhyang University

Hospital, 59

Daesakwan-ro, Yongsan-gu,

Seoul 04401 , Korea

Tel: $+82-2-709-9026$

Fax: $+82-2-709-9226$

E-mail: kblee@schmc.ac.kr
The objective of brain computed tomography (CT) in patients with hyperacute stroke is to exclude any intracranial hemorrhage, which is an absolute contraindication against thrombolysis. Hyperdense artery signs on brain CT have been correlated with large volume infarcts or severe neurological deficits, and may require an emergent intraarterial thrombectomy. ${ }^{1,2}$ While hyperdense middle cerebral artery (MCA) signs are frequently reported, hyperdense anterior cerebral artery (ACA) signs have rarely been described in the literature..$^{3-5}$ Hyperdense ACA signs show relatively low sensitivity and interobserver agreement, even in a meticulously designed retrospective study. ${ }^{1}$ Accordingly, hyperdense ACA signs are often ignored by emergency department physicians as well as stroke neurologists in the clinical practice. In addition, compared with hyperdense MCA signs, hyperdense ACA signs have fundamental limitations regarding their detectability on conventional axial brain CT scans. Considering the clinical implications, identifying hyperdense ACA signs is crucial for the diagnosis and immediate reperfusion therapy of hyperacute ACA infarctions. ${ }^{4}$ Here, we report a case of hyperdense ACA sign that was rapidly identi- fied using sagittal brain CT images.

\section{CASE}

A 49-year-old woman visited the emergency room at midnight, with an abrupt weakness in her left leg that had started 3.5 hours earlier. She showed hemiparesis (MRC grade 3), hemisensory loss, and ataxia on the left side on neurological examination. Her initial National Institute of Health Stroke Scale (NIHSS) score was 7. She had no history of medical illness or medication. Her initial vital signs were stable with a regular heartbeat. The results of laboratory tests for coagulopathy, vasculitis, and connective tissue disease were unremarkable. Echocardiography and Holter monitoring did not reveal any source of cardioembolism. Emergent brain CT was conducted 4 hours after stroke onset and showed a small hyperdensity with a radiodensity of 76 Hounsfield units (HU) in the interhemispheric fissure (Fig. 1A). To differentiate between an intraarterial thrombus and hemorrhage, sagittal images were rapidly reconstructed (Fig. 1C) and 
revealed string-like hyperdensities along the corpus callosum, which were suspected to be occluding thrombus from the A2 segment (Fig. 1B). Intravenous t-PA was immediately administered and revealed an acute right ACA infarction on subsequent diffusion-weighted imaging (Fig. 2A) and a distally migrated residual thrombus on susceptibility-weighted imaging (Fig. 2B). Timeof-flight magnetic resonance (MR) angiography showed recanalization of the A2 segment of the right ACA (Fig. 2C). The patient's neurological deficit was significantly improved, with an NIHSS score of 2, after intravenous thrombolysis.

\section{DISCUSSION}

Because of limited time and availability of MR imaging (MRI), CT is still the imaging modality of choice for patients with hyperacute stroke in the emergency room. The axial brain CT images of our patient revealed a small hyperdensity in the interhemispheric fissure. Due to increased local hematocrit caused by clotted cells and debris, thrombi have a higher absorption value $(\sim 8 \mathrm{OHU})$ than flowing blood ( $4 \mathrm{O} \mathrm{HU})$ on CT scans. Previous pathological studies revealed that hyperdense artery signs correlate with occluding thrombi., ${ }^{4,6,7} \mathrm{Em}$ bolic etiology is found in most cases of hyperdense ACA
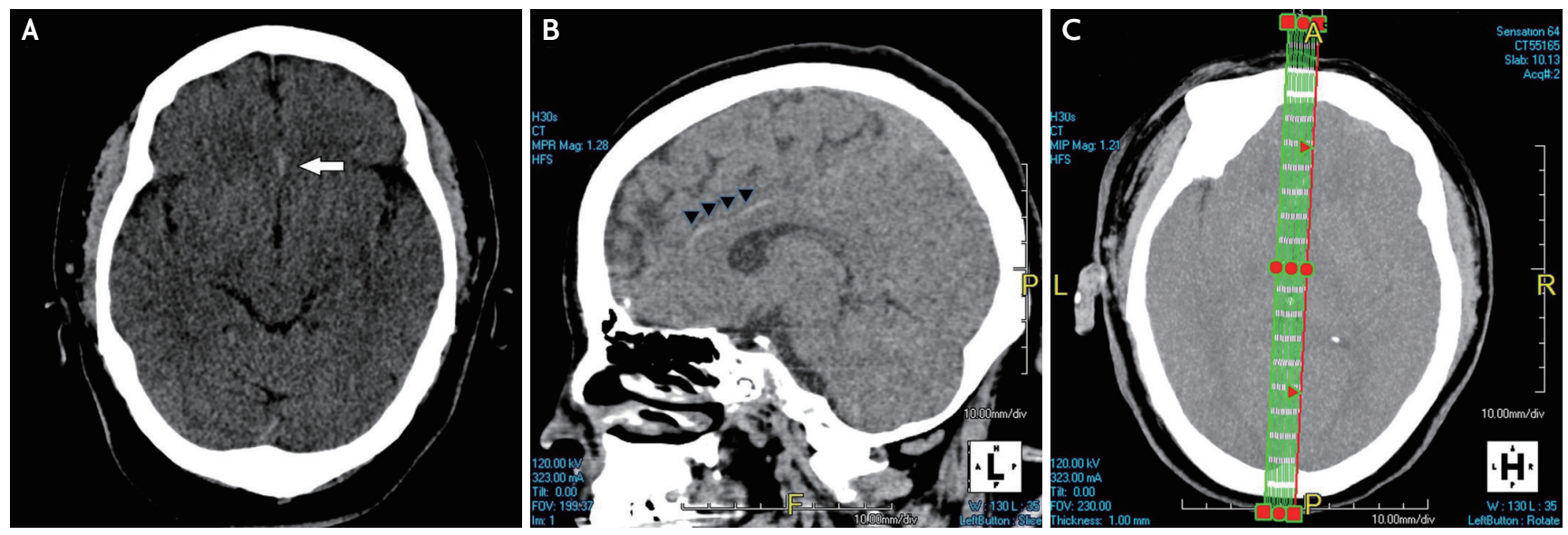

FIG. 1. (A) Brain computed tomography (CT) showing a small hyperdensity (white arrow) in the interhemispheric fissure on an axial scan, and (B) a long string-like hyperdensity (arrow heads) along the anterior cerebral artery on a sagittal scan. (C) Instant design of section on axial scan of CT for sagittal reconstruction.
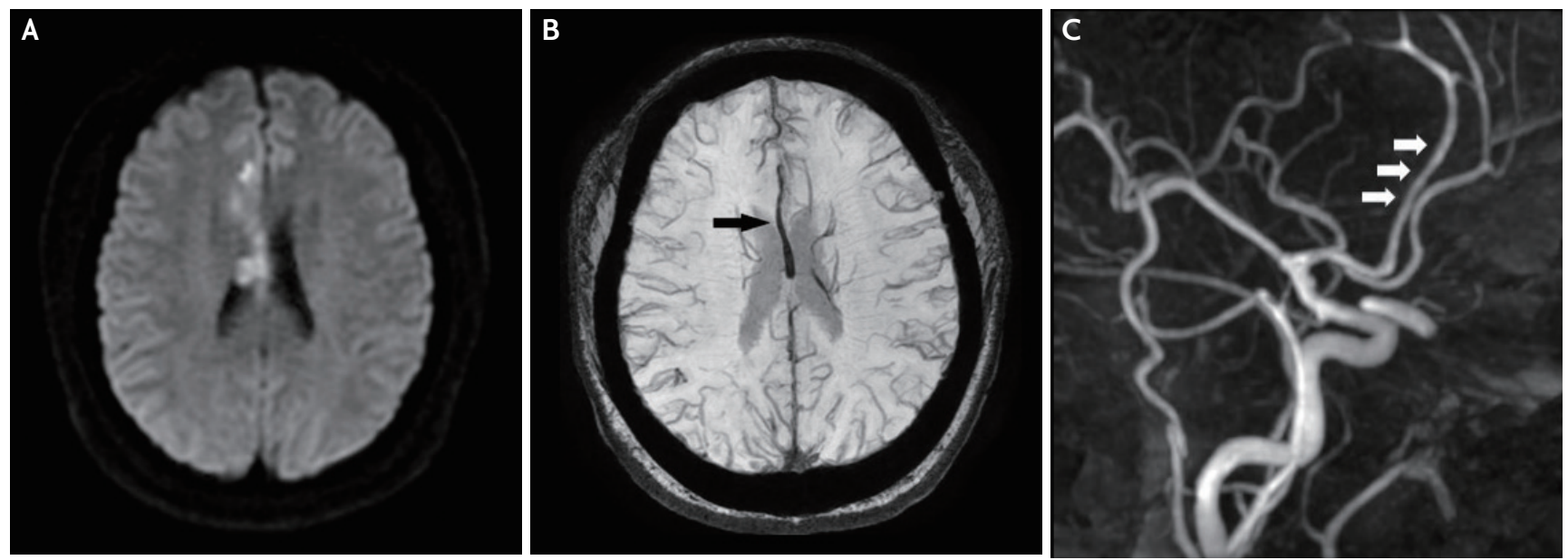

FIG. 2. (A) After intravenous thrombolysis, an acute infarct can be seen on diffusion-weighted images and (B) a residual thrombus (black arrow) on susceptibility-weighted images. (C) Time-of-flight angiography the next day shows the A2 segment of the right anterior cerebral artery (white arrows) almost completely recanalized. 
signs and presumed to be of cardiac, undetermined, or artery-arterial origin. ${ }^{4}$ Here, no cardioembolic source was identified, but the patient showed recanalized ACA suggesting embolic stroke of undetermined origin.

The anatomic course of the ACA in the A1 and proximal A2 segments runs parallel to the axial planes on brain CT. A hyperdense ACA sign in these relatively short segments is easy to detect because the in-plane resolution of CT is higher than its resolution along the Z-axis. ${ }^{4}$ Distally, both the pericallosal artery and the callosomarginal artery run perpendicularly to the axial planes when they wind around the corpus callosum, so that a hyperdense section might escape detection. The embolus of the patient in this study seemed to have been initially lodged in the A2 segment and then distally migrated. The diameter of the distal part of the ACA is considerably smaller than that of the MCA, which could explain the difficult detection of a hyperdense ACA sign. If the slice is much thicker than the affected vessel, the hyperdense vessel might not be visualized due to partial volume effects. In such a situation, sagittal planes can be superior to typical axial planes on brain CT in displaying the thrombus along the vertical path of the ACA. Axial thin-section CT (1.25- or 1-mm thickness) is also considered more sensitive for hyperdense artery signs than conventional CT at $\geq 5-\mathrm{mm}$ thickness. ${ }^{5}$

According to the current treatment guidelines, intravenous thrombolysis with t-PA infusion can be considered in hyperacute ischemic stroke within 4.5 hours from stroke onset. ${ }^{2}$ In our case, since brain CT was conducted 4 hours after stroke onset, we did not have time to conduct CT angiography in combination with the administration of a contrast agent via a larger intravenous needle within the therapeutic window of t-PA. Therefore, an urgent decision on how to differentiate between a thrombus and hemorrhage, such as an aneurysmal rupture of the anterior communicating artery, was needed, rather than CT angiography or brain MRI. By choosing sagittal brain CT, we prevented a delay in door-to-needle time, which is highly associated with unfavorable outcomes. ${ }^{8}$

To our knowledge, a hyperdense ACA sign identified on sagittal brain CT has not been depicted in the lit- erature. We thus suggest sagittal brain CT as a useful option to confirm a hyperdense ACA sign as well as immediate reperfusion therapy to avoid delays in door-toneedle time.

\section{Conflicts of Interest}

No potential conflicts of interest relevant to this article was reported.

\section{REFERENCES}

1. Jensen-Kondering U, Riedel C, Jansen O. Hyperdense artery sign on computed tomography in acute ischemic stroke. World J Radiol. 2010;2:354-357.

2. Jauch EC, Saver JL, Adams HP Jr, Bruno A, Connors JJ, Demaerschalk BM, et al. Guidelines for the early management of patients with acute ischemic stroke: a guideline for healthcare professionals from the American Heart Association/American Stroke Association. Stroke. 2013;44:870-947.

3. del Saz-Saucedo P, Maestre-Moreno JF, Vatz KA, Pérez-Navarro MJ, Fernández CC, Hernás-Navidad R, et al. Sign of hyperdense and hyperintense anterior cerebral artery. Neurologia. 2007;22:184-186.

4. Jensen UR, Weiss M, Zimmermann P, Jansen O, Riedel C. The hyperdense anterior cerebral artery sign (HACAS) as a computed tomography marker for acute ischemia in the anterior cerebral artery territory. Cerebrovasc Dis. 2010;29:6267.

5. Kim EY, Lee SK, Kim DJ, Suh SH, Kim J, Heo JH, et al. Detection of thrombus in acute ischemic stroke: value of thin-section noncontrast-computed tomography. Stroke. 2005;36:2745-2747.

6. New PF, Aronow S. Attenuation measurements of whole blood and blood fractions in computed tomography. Radiology. 1976;121 (3 Pt. 1):635-640.

7. Schuierer G, Huk W. The unilateral hyperdense middle cerebral artery: an early CT-sign of embolism or thrombosis. Neuroradiology. 1988;30:120-122.

8. Kamal N, Sheng S, Xian Y, Matsouaka R, Hill MD, Bhatt $\mathrm{DL}$, et al. Delays in door-to-needle times and their impact on treatment time and outcomes in get with the guidelines-stroke. Stroke. 2017;48:946-954. 